(C) 2007 The Japan Society of Applied Physics

\title{
Near-Field Optics Imaging in Silica Waveguide Using Near-Field Scanning Optical Microscope
}

\author{
Yuan-Fong $\mathrm{CHAU}^{*}$ and Din Ping TsaI ${ }^{1}$ \\ Department of Electronic Engineering, Ching Yun University, Jung-Li, Taiwan, R.O.C. \\ ${ }^{1}$ Department of Physics and Center for Nanostrage Research, National Taiwan University, Taipei, Taiwan, R.O.C.
}

(Received April 21, 2006; accepted September 21, 2006; published online January 10, 2007)

We investigated the near-field optics imaging by a numerical method using special spherical chains created in a silica waveguide (SWG) and explored the possibility of how this can be used for near-field scanning optical microscopy (NSOM) applications. There are two types of spherical nanochain; type A includes nano-dielectric spheres connected closely, and type B includes silver nanoparticles $4 \mathrm{~nm}$ in diameter randomly distributed inside the nanospheres of type A. A spherical nanochain with the 80-nm-diameter spheres was embedded in the SWG. The interactions between light emerging from SWG and the electric field near the sample surface that affects the properties of the images produced by NSOM are analyzed using a three dimensional finite-difference time-domain method. The effects of the optical field distribution generated by some factors are also discussed. A method of controlling the light field localized in the SWG is proposed that uses the external protrusion (dielectric sphere). The proposed structure will yield some useful information and give an idea of applications in NSOM. [DOI: 10.1143/JJAP.46.238]

KEYWORDS: silica waveguide, super-RENS, finite-difference time-domain method, scanning optical microscopy (NSOM), nanoparticles

\section{Introduction}

Near-field scanning optical microscopy $(\mathrm{NSOM})^{1-4)}$ is well known to be a powerful tool for the observation of microstructures beyond the diffraction limit. Optical information on near-field between the sample area and the output end of a silica $\left(\mathrm{SiO}_{2}\right)$ waveguide $(\mathrm{SWG})$ of nanometric dimensions that can be obtained from NSOM images has remained unclear, because the imaging process of NSOM is relatively complicated. A near-field electric field distribution is formed in SWG and diffracts owing to the optical properties of the sample such as geometrical structure, refractive index and absorption distribution. The light field localized in nanochains embedded in SWG as a defect region within areas in the near-field zone has never been studied to our knowledge. The field intensity distribution at the output end of the SWG is also sensitive to the presence of a probe object (external dielectric sphere), offering a way to control the light field localized in SWG. In our numerical simulation, a three-dimensional (3D) finite-difference timedomain (FDTD) ${ }^{5-8)}$ method with perfectly matched layer boundary conditions ${ }^{9)}$ is used to analyze the propagation of a light beam in the defect region of SWG. This analysis reveals several intriguing features of light localization in SWG, which seems to offer much promise for NSOM. In particular, subwavelength resolution can be achieved with SWG when the localization of a light wave occurs within areas whose sizes are less than a wavelength. This provides information concerning the spatial distribution of the electromagnetic field emerging from the output end of SWG.

In this paper, we focus on the change in near-field light for a model that we consider for SWG with two types of nanochain. The different aspects of the local interaction of the field distribution between SWG and the external sphere, and a sample with a protrusion (dielectric sphere) are taken into account. Firstly, we discuss some preliminary results recorded with the near-field distribution of such a subwa-

*E-mail address: yfc01@cyu.edu.tw velength structure for two types of nanochain as an extra defect inside SWG, and then a system including SWG and an external dielectric sphere is investigated. Finally, a novel idea of NSOM using an evanescent wave emerging from SWG through a sample with a protrusion is proposed, which seems to offer promising applications in NSOM.

\section{Simulation Models}

Figure 1 shows a schematic diagram of 3D SWG with two types of nonochain to be analyzed. The SWG considered here has a refractive index $n=1.5$, width $w=200 \mathrm{~nm}$, and a height $h=200 \mathrm{~nm}$, and the surrounding medium is air $(n=1)$. As shown in Fig. 1, two types of nanochain embedded inside the central part of SWG are illustrated. Type A includes four spherical chains connected closely with a refractive index $n=3.4$ (GaAs) and a diameter of $80 \mathrm{~nm}$. Type B includes the same chains as those in type A except for the silver nanoparticles embedded inside the dielectric spheres of type A. In the dielectric spheres of type B, silver nanoparticles $4 \mathrm{~nm}$ in diameter are randomly positioned in the 80-nm-diameter spheres, as shown in Fig. 1. The refractive index of each silver nanoparticle is chosen to be $0.7+i 3.65$. $^{10}$ The dispersive behavior of silver nanoparticles is simulated using the Lorentz model. ${ }^{11)}$ The effects of light polarization on the generated near-field images are studied under both parallel and perpendicular polarization conditions. When the model structure possesses a preferred orientation, the characteristics of the scattered light depend on the polarization direction (either parallel or perpendicular) relative to the structure orientation. In this paper, we report that the incident electric field is p-polarized when the electric field vector oscillates in a cross-sectional plane that is perpendicular to the surface of the edge structure (electric field $E$ along $x$ - or $y$-axis; transverse polarization). On the other hand, s-polarization occurs when the field vector oscillates in a direction normal to a crosssectional plane (electric field $E$ along the $z$-axis; longitudinal polarization).

Firstly, we observed the field distribution using the 3D 


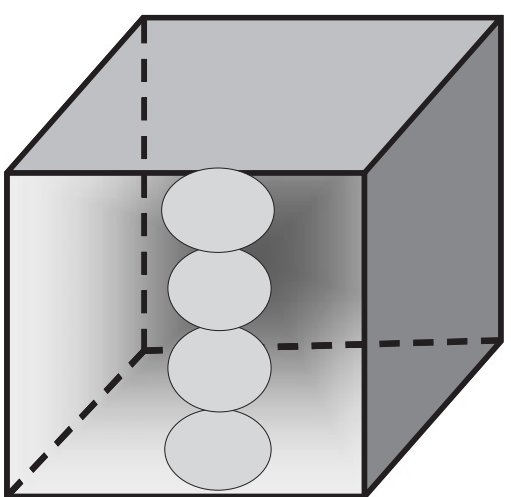

(a)
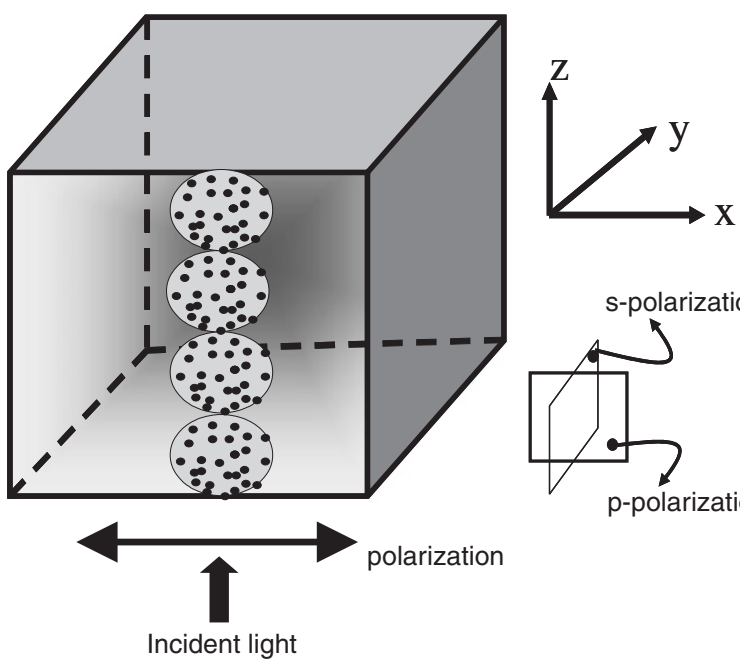

(b)

Fig. 1. Schematic diagram of 3D SWG with two types of spherical chain. The SWG considered here has a refractive index $n=1.5$, a width $w=200 \mathrm{~nm}$, a height $h=200 \mathrm{~nm}$, and the surrounding medium is air $(n=1)$. Two types of nanochain are embedded inside the central part of SWG. Type A (a) includes four spherical nanochains connected closely with a refractive index $n=3.4$ (GaAs) and a diameter of $80 \mathrm{~nm}$. Type B (b) is the same as type A except for the silver nanoparticles embedded inside the dielectric spheres of type A. In the dielectric spheres of type B, silver nanoparticles $4 \mathrm{~nm}$ in diameter are randomly positioned in the 80 nm-diameter spheres. The refractive index of each silver nano-particle is chosen to be $0.7+i 3.65$. $^{10}$

FDTD method inside SWG. The reason why we treat the spherical shape as an extra defect in SWG is that dielectric spheres can act as a lens and concentrate the light in the propagation direction. A p-polarized input Gaussian beam with a wavelength $\lambda=633 \mathrm{~nm}$ and implemented as a scattering field/total field source (SF/TF) ${ }^{5}$ is focused onto the central part of the spherical chain, located at the bottom face plane shown in Fig. 1 at a normal incidence. An other parameter that was used was cell size $\Delta x=\Delta y=\Delta z=$ $2 \mathrm{~nm}$, and the total space volume considered consists of $100(x) \times 100(y) \times 200(z)$ cells. The time step is chosen to be $\Delta t=0.95 / c\left(\Delta x^{-2}+\Delta y^{-2}+\Delta z^{-2}\right)^{-1 / 2}$, where $\mathrm{c}$ is the speed of light. The incident Gaussian beam's FWHM was adjusted such that FWHM $=0.51 \lambda / N A$.

\section{Results and Discussion}

Figures 2(a) and 2(b) show the calculated results of types $\mathrm{A}$ and $\mathrm{B}$, respectively. In the cross-sectional image plane shown in Fig. 2(b), highly localized enhancements were produced between adjacent silver nanoparticles in the nearfield zone. For an incident beam below the nanochain and an electric field $E$ perpendicular to the nanochain (p-polarization, wave vector $k$ will be along $z$-axis), free electrons of silver nanoparticles inside the nanochain oscillate collectively in resonance with the incident beam; the interaction may constitute surface plasmon polaritons (SPPs). The induced surface charge density is proportional to the discontinuity of the electric field component normal to the surface; only p-polarized light can excite SPPs, because incident beam with s-polarization ( $E$ along $z$ and wave vector $k$ will be in $x-y$ plane) can excite a very small intensity of evanescent wave contribution. These near-field optical signals of silver nano-particles have non-linear nearfield optical properties, which were previously found in experiments. ${ }^{12)}$ In the cross-sectional image of type A shown in Fig. 2(a), the field intensity is stronger both in the central part and near the rims of spherical chains (edge enhancement) than in other regions, and the image generated agrees reasonably with the geometrical profile of the same dielectric spheres. At the edge interface of the dielectric spheres, the electric flux density is conserved such that the electric field amplitude is greater than that in the outside medium where the permittivity is smaller $\left(\mathrm{SiO}_{2}, n=1.5\right)$. Note that the intensity in Fig. 2(b) contrasts with that in the image shown in Fig. 2(a). Silver nanoparticles are generated either in the sputtering process or by a reversible chemical reaction, and produce a large nonlinear optical effect. This effect arises from the excitation of plasmon resonances between the metallic particles (shape-dependence) that are embedded inside the dielectric spheres by the evanescent photons and leads to light wave propagation mainly in the forward direction. Under such a condition, one also expects the silver nanoparticles to serve as the local source of light if the incident electric field has a vertical component ( $p$ polarization). Another significant result from Fig. 2(b) is the effect of the embedded silver nanoparticles that form very strong scattering centers and increase the evanescent field. The embedded silver nanoparticles in the nanochain provide an interpretation for the high-scattering property of type B structure, which further enhances the surface plasmon effect and introduces nonlinear optical properties. It also provides strong local field enhancement, which may be of relevance to near-field optical processing.

As a result, the characteristics of an NSOM image of the surface are quite sensitive to the manner in which the probe is scanned over the sample surface. We obtained the spatial distribution of the optical field localization effect for the type B structure by controlling the external probe object (in our case, a dielectric sphere is used to imitate a local excitation source of particles). Figures 3(a) and 3(b) show the field intensity images obtained in the $x-z$ (at $y=0)$ and $x-y($ at $z=0)$ cross-sectional planes, respectively, when a transparent dielectric sphere with $n=1.5$ and the radius $r=40 \mathrm{~nm}$ [Figs. 4(a) and 4(b)] and $r=60 \mathrm{~nm}$ [Figs. 4(c) and $4(d)$ ] is located close to the output end of SWG, with the center of this sphere being placed at distances from the output facet of the SWG $d=20 \mathrm{~nm}$ [Figs. 3(a) and 3(b)] and $d=40 \mathrm{~nm}$ [Figs. 3(c) and 3(d)]. The dielectric sphere located at the output end of the SWG can be regarded as the local excitation source of the particle. A comparison of 

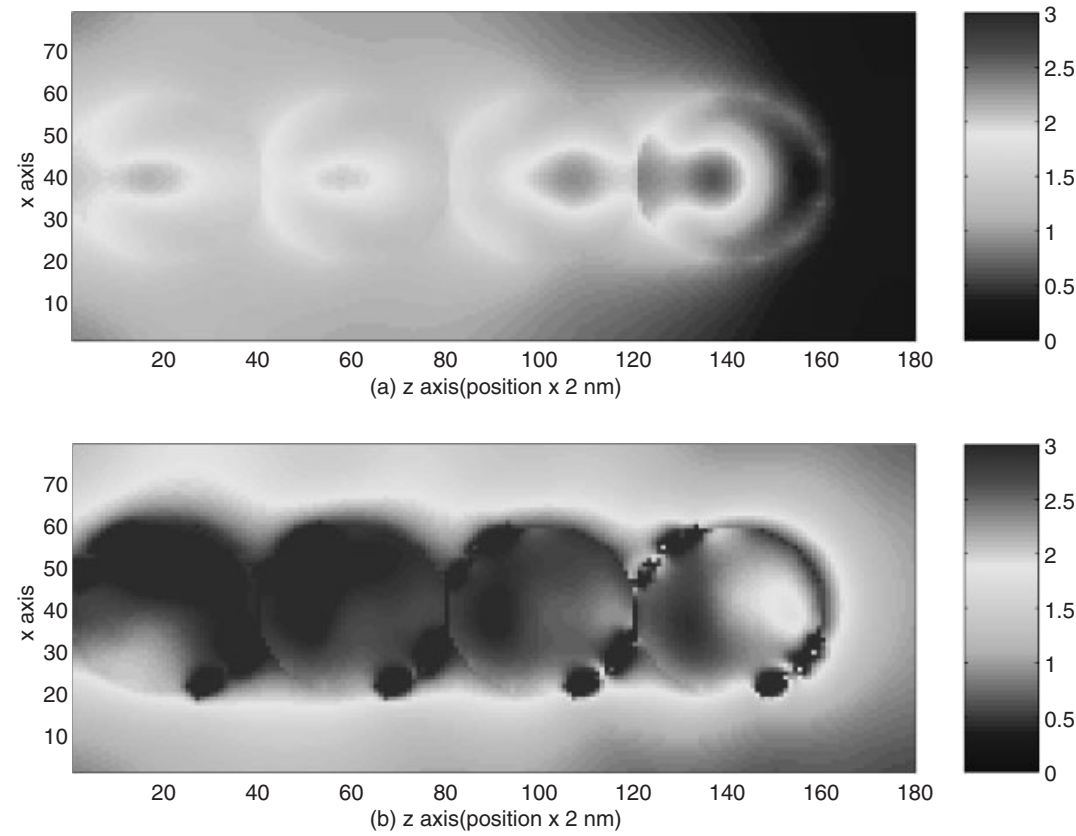

(a)

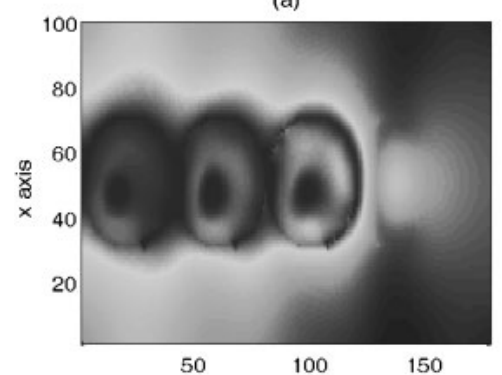

(c)

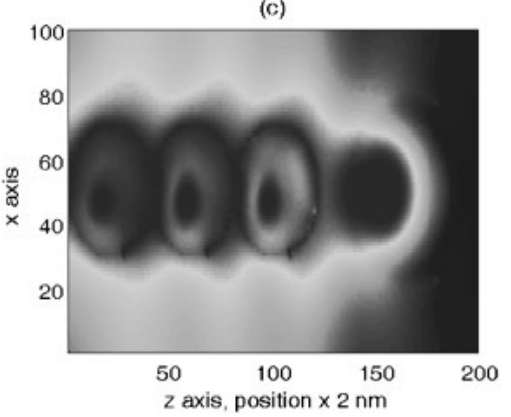

(b)

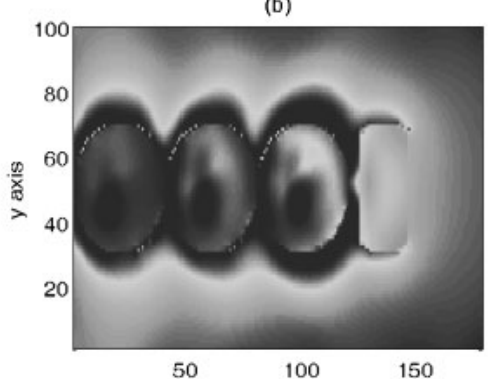

(d)

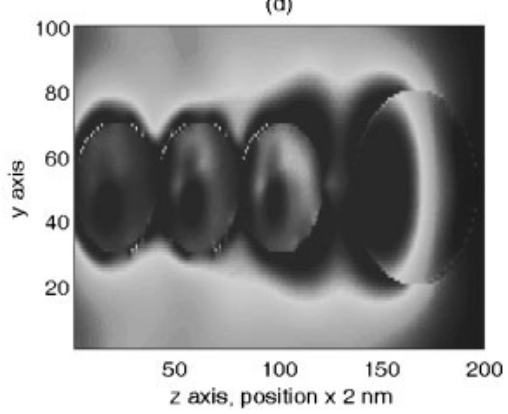

Fig. 2. Comparison of $x-z$ cross-sectional plane maps of light localization (field intensity distribution) of types A (top) and B (bottom).
Fig. 3. Results of calculated intensity images of type B obtained in $x-z$ (at $y=0)$ and $x-y($ at $z=0)$ cross-sectional planes for p-polarization illumination when transparent dielectric sphere with constant refractive index $n=1.5$ and radius $r=40 \mathrm{~nm}$ [(a) and (b)] and $r=60 \mathrm{~nm} \mathrm{[(c)} \mathrm{and} \mathrm{(d)]} \mathrm{is} \mathrm{located} \mathrm{close}$ to output end of SWG, with center of this sphere being placed at distances from output facet of SWG of $d=20 \mathrm{~nm}[(\mathrm{a})$ and (b)] and $d=40 \mathrm{~nm} \mathrm{[(c)} \mathrm{and}$ (d)].
Fig. 3 with Fig. 2(b) shows that the presence of such a dielectric sphere disturbs the field distribution inside the spherical chains, resulting in a phenomenon tunable by the potential of the spherical probe. This effect is due to a change in the disturbance source.

To determine the detailed behavior of the field distribution inside the nanochain, the three components of the field distribution will be discussed here. Figures 4(a)-4(c) show the $x-z$ cross-sectional plane maps of the field intensities of the three electric field components $\left(E_{x}, E_{y}, E_{z}\right.$ from top to bottom) for the cases shown in Fig. 3(c), where the image color scales are 0-4 for $E_{y}$ and 0-1 for $E_{x}$ and $E_{z}$. It can be observed in Fig. 4 that $E_{y}$ propagates mainly in the forward direction and forms the main field distribution of the total electric field emerging from SWG. The distance between SWG and the outer sphere decreases as $d$ value increases. The light intensity in SWG can be reduced, and the transmission light from the outer sphere is weaker. That is the light converted in the near-field zone is transmitted less to a photodetector than to the light between SWG and the outer sphere. The physically observable effect offers a better understanding that will lead to an unambiguous analysis of an NSOM image and will make NSOM a more reliable imaging tool for possible applications of the light localization effect in SWG or in biomedical and materials sciences. For example, it caninduce local excitation, and can be detected by a near-field fiber tip placed in the near-field zone.

As shown in Fig. 2(b), type B was used as an extra defect region inside $S W G$, and the field enhancement is anticipated with the local mode of the SPPs. We demonstrate these merits of NSOM. We combine line defects (spherical nanochains) and point defects (silver nano-particles) to make a novel SWG for NSOM application. The surface 
(a)

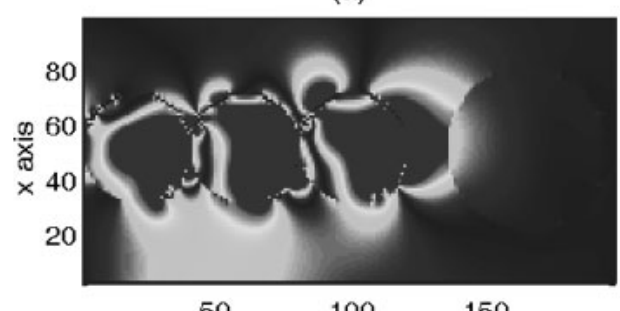

(b)

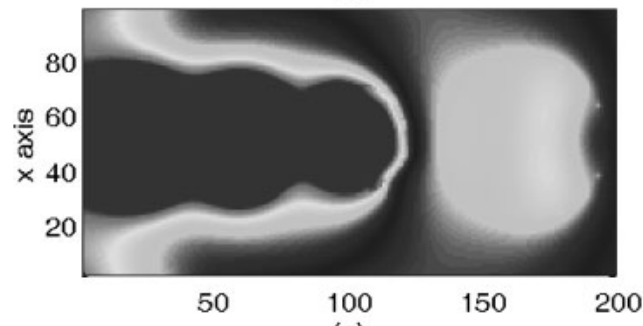

(c)

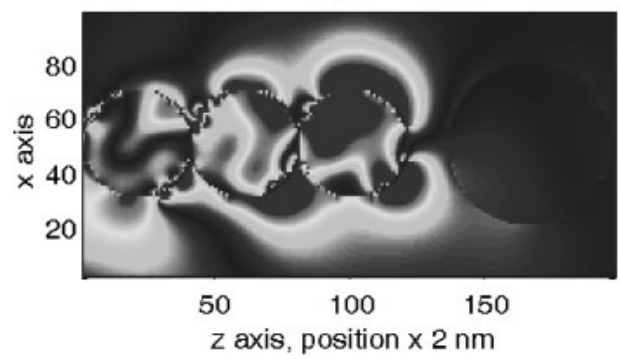

profile of the dielectric sample $(n=1.5)$ consists of a flat surface of thickness with a $15 \mathrm{~nm}$ with a dielectric spherical protrusion (used to imitate the tip of near-field fiber probe, as described in our previous works ${ }^{13,14)}$ ) whose diameter $d=60 \mathrm{~nm}$ and refractive index $n=1.5\left(\mathrm{SiO}_{2}\right)$ as shown in Fig. 5. SWG is illuminated normally by an incident beam with p-polarization from below and the light intensity of the sample on the surface facing the probe is detected in the far-field region (collection-type NSOM). Once the incident beam is inside SWG, it has nowhere else to go when one channel (spherical chain) is dropped at one carrier wavelength. The intensity of the electric field distribution in $x-z$ and $x-y$ cross-sectional planes, whose fragment is shown in Fig. 5, is presented in Fig. 6. It can be observed in Fig. 6 that the light beam is channelled along the forward propagation direction and the field is localized at the center and rims of the spherical chains. The interaction between the SWG and the field near the sample surface is an important mechanism in the formation of NSOM images. When the effects of multiple scattering by a dielectric spherical protrusion on the sample are taken into account, the sample, SWG and dielectric spherical protrusion can be regarded as one system in image formation. The evanescent field propagating through the dielectric spherical protrusion can transform to a radiation wave and be received by a photodetector. The effect of multiple scattering or image formation becomes negligible between SWG and the sample. The image is generated primarily by the coupling effect of the near-field to the waveguide structure of SWG. The sample divided the light emerging from the SWG into two parts; one penetrates through the sample, the other distributes between the sample and SWG. The light penetrating through the sample can be detected by a
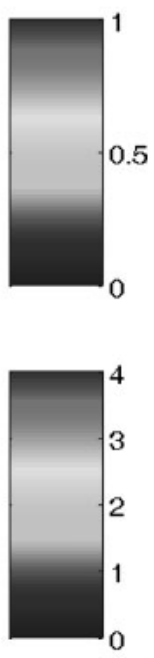

Fig. 4. The $x-z$ cross-sectional plane maps of three electric field components $\left(E_{x}, E_{y}, E_{z}\right.$, from top to bottom) corresponding to cases shown in Fig. 3(c), where image color scales are $0-4$ for $E_{y}$ and $0-1$ for $E_{x}$ and $E_{z}$.

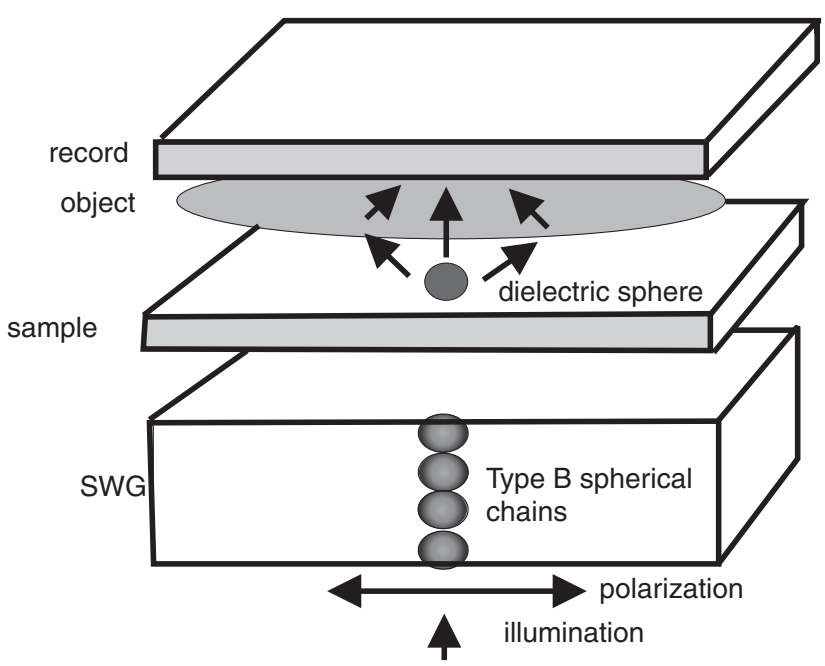

Fig. 5. Fragment of near field optical microscopy using evanescent wave formed in nanochain in SWG. The SWG structure with a defect (type B) is used to excite the evanescent wave with recording in the far field.

photodetector. The photodetector not only detects how the field distributes between SWG and the sample surface but also detects the light emerging from SWG, including the light refracted from the sample surface distributed between SWG and the sample. Hence, the generated NSOM intensity image exhibits a strong intensity on the rim of the spherical chains and on the flat sample surface. Field intensities larger than that of the SWG-sample interaction are not included. The light propagates out of the sample surface and creeps latterly along the sample surface acting with the characteristics of reflected light. On the rim of a spherical nanochain 
(a)
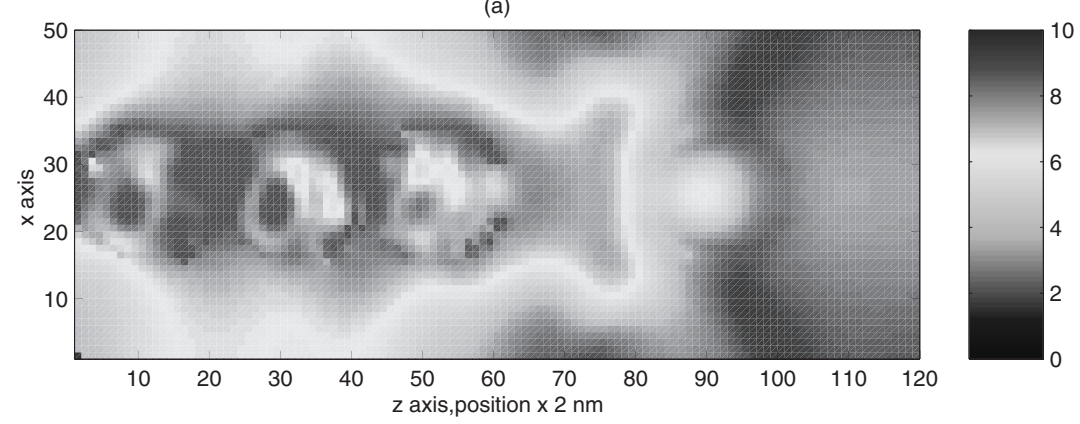

(b)

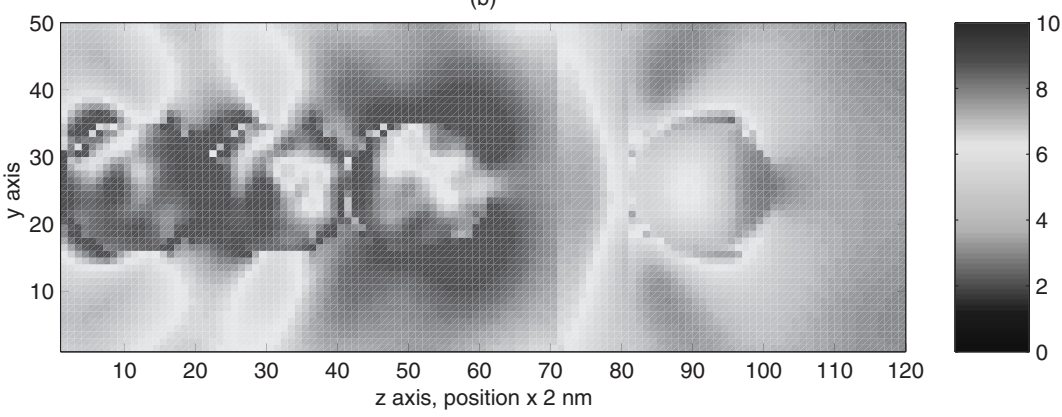

Fig. 6. 3-D electric field distributions of mean intensity of electric field in $x-z$ (at $y=0)$ and $x-y$ (at $z=0$ ) cross-sectional planes calculated for structure shown in Fig. 5. in SWG, multiple scattering occurs inside SWG and the intersection area between the sample and SWG, but is not coupled with the propagation mode of the fiber tip transmission.

The applications of the proposed SWG to NSOM and its advantages over conventional NSOM will be discussed here. The proposed NSOM offers a higher spatial resolution and sensitivity than the scattering-type NSOM owing to the SPP effects arising from the silver nanoparticles which are randomly positioned inside the type B structure. The effects of embedded silver nanoparticles are that they generate stronger scattering centers and a greater evanescent field than those in conventional NSOM. Silver nanoparticles enhance the role of local enhanced light, which provides a stronger field intensity and further enhances the SPP effects. The 80-nm-diameter dielectric spherical nanochain can be regarded as a confocal lens that concentrates light in the propagation direction ( $z$-direction). In addition, a type B structure shows both point defects (silver nano-particles) and line defects (dielectric spherical nanochains) in SWG, which may be relevant for offering promising applications in NSOM. In the near future, we intend to consider other effects involving the nonlinear response of the irradiated sample, e.g., the NSOM application using photonic crystals (to position such a structure (type B) in a photonic crystal waveguide) to near-field optical image processing.

\section{Conclusions}

In conclusion, we presented numerical results for calculating the near field distributions between the light field of a nanochain emerging from SWG and the light field near the sample surface in NSOM using a 3D FDTD method. We used two types of spherical nanochain embedded in SWG. The calculation results show that the evanescent fields formed in SWG can be used in the NSOM system with a spatial resolution exceeding the wavelength of the incident illumination. The high density of the optical field at the output end emerging from SWG may give rise to physically observable phenomena in the near-field zone. Many applications using these properties arising from the field at the output end of SWG are associated with the chances of possibilities the spatial resolution in photolithography, promoting the data storage capability in optical memory systems and its use as functional building blocks for nanoscale waveguiding devices, sensors and optoelectronics, and in controlling atom and atom interactions in quantum calculation problems, as well as in other applications.

\section{Acknowledgement}

The authors gratefully acknowledge the financial support from the National Science Council, Taiwan, R.O.C., under grant number NSC 95-2112-M-231-001, and the Ministry of Economic Affairs, Taiwan, R.O.C., under grant number 95EC-17-A-08-S1-0006.

1) D. W. Pohl and D. Courjon: Near Field Optics (Kluwer, Dordrecht, 1993) NATO ASI Series E, Vol. 242.

2) E. Betzig, M. Isaacson, and A. Lewis: Appl. Phys. Lett. 51 (1987) 2088.

3) D. W. Pohl, W. Denk, and M. Lanz: Appl. Phys. Lett. 44 (1984) 651.

4) G. Krausch, S. Wegscheider, A. Kirsch, H. Bielefeldt, J. C. Meiners, and J. Mlynek: Opt. Commun. 119 (1995) 283.

5) A. Taflove: Computational Electrodynamics: The Finite-Difference Time-Domain Method (Artech House, Norwood, MA, 2000) 2nd ed.

6) S. A. Maier, P. G. Kik, and H. A. Atwater: Appl. Phys. Lett. 81 (2002) 1714.

7) L. A. Sweatlock, S. A. Maier, H. A. Atwater, P. C. S. Amsterdam, and A. Polman: Phys. Rev. B 71 (2005) 235408.

8) W. A. Challener, I. K. Sendur, and C. Peng: Opt. Express 11 (2003) 3160 .

9) J. P. Berenger: J. Comput. Phys. 114 (1994) 185.

10) T. C. Chu, W. C. Liu, and D. P. Tsai: Opt. Commun. 246 (2005) 561.

11) J. B. Judkins and R. W. Ziolkowski: J. Opt. Soc. Am. A 12 (1995) 1974.

12) T. Nakano, A. Sato, H. Fuji, J. Tominaga, and N. Atoda: Appl. Phys. Lett. 75 (1999) 151.

13) Y. F. Chau, T. J. Yang, and D. P. Tsai: Jpn. J. Appl. Phys. 43 (2004) 8115.

14) Y. F. Chau, T. J. Yang, and D. P. Tsai: J. Appl. Phys. 95 (2004) 3378. 